\title{
Urgences
}

\section{Les manuscrits flaubertiens ou la recherche de l'absolu}

\section{Jeanne Goldin}

Numéro 24, juillet 1989

Le manuscrit sous l'angle

URI : https://id.erudit.org/iderudit/025536ar

DOI : https://doi.org/10.7202/025536ar

Aller au sommaire du numéro

Éditeur(s)

Urgences

ISSN

0226-9554 (imprimé)

1927-3924 (numérique)

Découvrir la revue

Citer cet article

Goldin, J. (1989). Les manuscrits flaubertiens ou la recherche de l'absolu.

Urgences, (24), 95-102. https://doi.org/10.7202/025536ar d'utilisation que vous pouvez consulter en ligne.

https://apropos.erudit.org/fr/usagers/politique-dutilisation/ 


\section{Les manuscrits flaubertiens ou la recherche de l'absolu}

\section{Jeanne Goldin, Université de Montréal}

Les avant-textes flaubertiens sont exemplaires comme le sont les travaux d'Alberto Cento, de Giovanni Bonaccorso, de l'équipe du C.A.M. (Centre d'histoire et d'analyse des manuscrits modernes) et bien d'autres. Les uns et les autres exemplaires autant par leur complexité que par l'intensité du Désir qui les sous-tend.

En ce qui concerne l'avant-texte de Madame Bovary, si l'on s'en tient au seul manuscrit des brouillons, il compte près de 1800 feuillets, la plupart écrits recto-verso, pour un roman dont vous connaissez l'épaisseur; et 329 to, pour le seul chapitre des Comices agricoles. ${ }^{1}$

J'y ai tous mes personnages [...] en action et en dialogue, les uns mêlés aux autres, et par là-dessus un grand paysage qui les enveloppe. Mais si je réussis, ce sera bien symphonique $[\ldots]^{2}$ J'ai un fouillis de bêtes et de gens beuglant et bavardant avec mes amoureux en dessus [...]. ${ }^{3}$ II faut que ça hurle par l'ensemble, qu'on entende à la fois des beuglements de taureaux, des soupirs d'amour et des phrases d'administrateurs. II y a du soleil sur tout cela et des coups de vent qui font remuer les grands bonnets. ${ }^{4}$

L'effet "symphonique" recherché par Flaubert dans ce chapitre justifie les cinq mois de travail acharné qu'il lui a consacrés, du 15 juillet au 9 décembre 1853.

Passons rapidement sur le capharnaüm du Ms G 223: la lisibilité relative, le désordre du manuscrit tel qu'il se présente à la bibliothèque de Rouen, la pagination de Flaubert inopérante dans l'immédiat, la discontinuité diégétique du recto au verso, les

1 Jeanne Goldin, Les Comices agricoles de Gustave Flaubert. Transcription intégrale et genèse dans le manuscrit G 223, Paris, Droz, 1984,2 vol.

2 Gustave Flaubert, Correspondance, Paris, Louis Conard, 19261954, III (A Louise Colet, 7 septembre 1853).

3 Ibid., 21 septembre 1853.

4 Ibid., 7 octobre 1853. 
découpages et les collages maintenant dispersés. La résolution de ces problèmes est préalable à l'inventaire et à l'ordonnance diégétique et génétique des feuillets, de même que cette ordonnance est la condition sine qua non de toute analyse.

Le paragraphe 100 des Comices, que j'ai choisi parmi les moins problématiques pour vous en présenter la genèse, peint Lieuvain appelant l'attention de son auditoire et celui-ci figé dans la fascination, la somnolence béate ou l'accablement - ce qui m'a semblé convenir particulièrement à la fin d'un colloque.

II n'avait pas besoin de l'appeler: car toutes les bouches de la multitude se tenaient ouvertes, comme pour boire ses paroles. Tuvache, à côté de lui, l'écoutait en écarquillant les yeux; $M$. Derozerays, de temps à autre, fermait doucement les paupières; et plus loin, le pharmacien, avec son fils Napoléon entre les jambes, bombait sa main contre son oreille pour ne pas perdre une seule syllabe. Les autres membres du jury balançaient lentement leur menton dans leur gilet, en signe d'approbation. Les pompiers, au bas de l'estrade, se reposaient sur leurs baïonnettes; et Binet, immobile, restait le coude en dehors, avec la pointe du sabre en l'air. II entendait peut-être, mais il ne devait rien apercevoir à cause de la visière de son casque qui lui descendait sur le nez. Son lieutenant, le fils cadet du sieur Tuvache, avait encore exagéré le sien; car il en portait un énorme et qui lui vacillait sur la tête, en laissant dépasser un bout de son foulard d'indienne. II souriait là-dessous avec une douceur tout enfantine, et sa petite figure pâle, où des gouttes ruisselaient, avait une expression de jouissance, d'accablement et de sommeil.

Giovanni Bonaccorso, dans son édition de l'avant-texte d'Un cœur simple, distingue 8 stades génétiques: 1. le plan; 2 . les notes; 3 . le scénario; 4 . le résumé; 5 . l'esquisse; 6 . le brouillon; 7 . la mise au net; 8 . l'état final.

Les cinq premiers stades visent, sous une forme elliptique, la recherche des idées et/ou la structuration de grands pans du récit. Le Ms G 9 édité par Jean Pommier ${ }^{5}$ est constitué de textes de ce genre (quoique le Ms G 223 des brouillons contienne nombre de notes, d'esquisses et de résumés inédits). Notre paragraphe 100 est trop modeste pour y figurer comme tel; mais évidemment il y prend place, d'abord négativement

5 Jean Pommier et Gabrielle Leleu, Madame Bovary. Nouvelle version précédée des scénarios inédits, Paris, José Corti, 1949. 
dans les plans généraux et les scénarios, chaque fois que l'on y parle des Comices, et en particulier, lorsque Flaubert insiste sur la nécessité de mêler les différents plans du récit: “rappeler les personnages secondaires [...] Lier les Comices et le dialogue"; ensuite indirectement, à l'occasion d'autres séquences ou dans des notes sur les motifs qui le constituent. Par exemple, lors de l'arrivée des Bovary à Yonville, une note de Flaubert sur Binet semble annoncer sa silhouette dans les comices et ses ennuis avec les couvre-chefs: "Le percepteur: [...] crâne qui a porté le casque [...] toute petite casquette [...]" (SC.26). Enfin directement, une addition à la description générale du début des Comices sembler anticiper notre paragraphe: "aspect général [...] garde nationale au-devant pompiers - Binet, mouchoirs dans les casques" (SC.39).

Mais notre passage ne naît véritablement qu'en cours de rédaction et c'est dans le manuscrit $G 223$ des brouillons que l'on peut en suivre la croissance, sur huit pages: trois esquisses (fo $131 / 265$; fo $137 / 278$; fo $139 / 278$ ) et cinq réécritures (fo [b.149 bis $/ 283+$ b. $\left.174 \mathrm{~V} / /^{\star}\right]$; fo [ b. $175 \mathrm{~V} / 284+$ b. $\left.146 \mathrm{~V} / /^{\star}\right]$; fo $173 \mathrm{~V} / /^{\star}$; b.174/284; fo $198 \mathrm{~V} / 284$ ). Dans mes transcriptions, un cadre de référence ajouté précise la double pagination (ex. fo 131/265): la première marquant l'ordre de classement et de reliure de la bibliothèque (ex. 131); la seconde, éventuelle, étant celle de Flaubert (ex. 265). Décrivons brièvement cette croissance.

Les trois premiers états (fo 131,137 et 139) sont trois esquisses qui structurent la partie centrale des Comices. Flaubert en effet a vu assez rapidement que, pour créer l'effet symphonique recherché, il devait procéder par feuilletages. Feuilletage du discours de Lieuvain (d'abord écrit en un seul bloc puis successivement découpé en cinq, six, huit, sept sections) et des lieux communs de Rodolphe et d'Emma dont la nature et les couches varient en proportion. Feuilletage également du discursif (les dialogues, les discours, l'article d'Homais) et du narratif/descriptif. La nature et la place de notre paragraphe 100 se présentent donc comme une conséquence logique à la fois du discours de Lieuvain et de l'intention flaubertienne de mêler des descriptions aux discours.

Dans la marge du fo 131, l'on voit ainsi naître le paragraphe 100 , dans les deux motifs flottants du fils Tuvache et d'Homais séparés par d'autres passages descriptifs. Dans l'esquisse 137, ils trouvent leur place avant l'«absorption»d'Emma au $\S 103$ et 
la conclusion du discours de Lieuvain, c.-à-d. à peu près comme dans le texte final. Quant à l'esquisse suivante 139 qui, travaillant sur le feuilletage discursif, ne les inclut pas, elle montre que la place de cette description dépend étroitement de la structuration générale.

Les cinq feuillets suivants sont, à proprement parler, des brouillons. Ils placent peu à peu le thème et les différents personnages de l'auditoire: la multitude, Tuvache, Derozerays, Homais, les membres du jury. (qui n'apparaissent que tardivement), les pompiers et Binet, enfin le fils Tuvache. Ni le quatrième ni le cinquième états ne semblent vraiment des mises au net. Le fo $198 \mathrm{~V} / 284$ est, en tout cas, le dernier brouillon que nous possédions, son texte, malgré ses ratures, étant le plus proche du texte de base. Les manuscrits 221 et 222 non exempts de corrections mineures font la transition avec le texte de base.

Je ne donnerai qu'un exemple d'analyse génétique; il concerne la circulation des motifs. Le paragraphe 100 , en effet, est en liaison implicite avec d'autres séquences.

D'abord, il n'est pas étranger à un passage au début des Comices que Flaubert avait travaillé, sur 18 fo, pour finalement le supprimer. La foule y regardait, avec une admiration croissante, un homme poser des lampions sur la façade de la mairie. Dans de nombreuses variantes, on voyait la multitude ébahie, fascinée, «les regards [...] comme des lampions, sur la façade" et finalement un long cri sortait "de toutes ces bouches béantes". Emma aussi sera bouche bée devant Rodolphe. Flaubert n'aura garde de perdre cette similitude et récupérera les bouches béantes à la première rédaction du paragraphe 100; biffé dans le même fo, le trait passe à Tuvache qui, bouche ouverte, se délecte profondément; les bouches béantes de la multitude reviennent à l'état suivant (fo $\left[\mathrm{b} .175 \mathrm{~V} / 284+\mathrm{b} .146 \mathrm{~V} /{ }^{*}\right]$ ) et, du même coup, Flaubert hésite à peindre Tuvache ouvrant la bouche et ouvrant les yeux en même temps. Dans la troisième réécriture, les bouches de la foule seront ouvertes et Tuvache écarqulllera les yeux.

Plus loin, la description de Binet renvoie au paragraphe 1 où Flaubert avait peint le personnage en parade, le buste immobile et raide et concentré tout le mouvement sur les jambes marquant la cadence. Dans une variante (fo 66/261) supprimée (fo 67/260), apparaissait "la visière de son casque qui lui 
descendalt sur le nez", qui sera récupérée à la première rédaction du paragraphe 100 (fo [b.149 bis + b.174V]).

Enfin, il est instructif de suivre, dans le détail, le motif du fils Tuvache. II apparaît au fo 131, mais d'abord inclus avec son père dans la description antérieure des bourgeois sur l'estrade. L'avant-texte de ce paragraphe 71 travaillera beaucoup les portraits du père et du fils se ressemblant malgré leurs différences; il montrera le maire avec ses "favoris blonds comme les blés, sa face rougeaude et satisfalten; et malgré leur jeune âge, ses fils "nlals, minces comme des perpignans" (fo 131), "endormis". (fo $130 / 277$; fo $124 / 276$ ) et ayant "la même expression tranquille de douceur artificieuse et de sensualité médiocre" (fo $94 \mathrm{~V} / 276$ ). Tous ces détails disparaîtront du paragraphe 71 , centré dès lors sur la similitude des attitudes et des mises; mais Flaubert les récupérera pour le paragraphe 100 , où les mêmes traits jouent à la chaise musicale. C'est Tuvache, au fo $175 \mathrm{~V}$, "la mine importante" et semblant "jouir profondément"; avec, au fo 173V, dans une variante biffée, récupérant la bêtise du fils:

l'invariable sourire qui dilatait sa large mine [...] statue de la bétise [...] il s'en estimait davantage et balançait dans son fauteuil avec son écharpe tricolore, sa mine importante et sa rotondité.

Son balancement sera récupéré par les membres du jury, seulement lors de la troisième réécriture (fo 173V). Simultanément, le portrait du fils Tuvache s'étoffe. Au casque, au foulard d'indlenne, à la douceur qui resteront des constantes de la première esquisse au texte de base, s'ajoutent peu à peu ses attributs personnels: à la première rédaction (fo [b.149 bis + b. $174 \mathrm{~V}]$ ), les yeux bleus, l'air endormi, et accablé; à la seconde (fo [b.175V + b.146V]), la jeunesse, le teint pále. Mais à chaque rédaction, des traits flottent entre le père et le fils: à la première, le sourire, à la seconde, le contentement, à la troisième (fo 173V), la jouissance. Lors de cette troisième réécriture, Flaubert récupère provisoirement, au profit du fils Tuvache, l'enivrement du soleil de la première esquisse (fo 131), mais en les dissociant. La quatrième rédaction (b.174/*) supprime les yeux bleus du fils, remplace sourire par regarder jusqu'au Ms 222 qui reviendra à sourire. Enfin, le dernier état confirme le tout, à quelques détails près.

Ces exemples montrent d'abord l'extrême dépendance de toute unité scripturale vis-à-vis de l'ensemble, leitmotiv de la 
poétique flaubertienne et le balayage constant que Flaubert fait de son texte: la moindre suppression crée une réaction en chaîne et libère un matériel qui se réinvestira en partie dans de nouveaux équilibres instables. Tout se tient, les feuillets saturés, le moindre bout de papier utilisé: volonté bourgeoise de thésaurisation, peur sordide et grandiose de toute perte, conscience vertigineuse de l'infini du langage et du monde. Le vertige de Flaubert qu'atteste sa correspondance ne peut que gagner celui qui marche dans les traces de l'avant-texte. Comme Flaubert, il prend conscience, en même temps, de son Désir et de son Impuissance.

J'ai travaillé plus de dix ans sur l'avant-texte d'un chapitre de Madame Bovary et les quelques réflexions qui vont suivre ne concluent qu'une expérience personnelle. Je voulais en effet éclaircir, pour moi, le paradoxe suivant: autant mon expérience des manuscrits m'a été essentielle, autant, je l'avoue, toute étude génétique ne m'est accessible que très superficiellement: si l'on étale toutes les données, je suis accablée par leur masse, suspicieuse sur leur établissement et perds le fil d'Ariane; si, par contre, l'on élude les jalons, si l'on synthétise les conclusions, toujours déçue, je n'y trouve qu'un intérêt général, disproportionné à l'énergie mise en œuvre.

Or, cette impression subjective me semble pourtant liée à la nature même des études génétiques (au moins flaubertiennes), leur tension entre la scientificité qu'elles recherchent et la mystique qui les informe. J'ai accordé ici plus d'importance à la première (l'ACFAS oblige!), en présentant l'avant-texte d'un paragraphe de Madame Bovary et en faisant ainsi une démonstration par l'absurde; mais, en fin de course, déplaçant l'intérêt des résultats laborieusement conquis à la démarche elle-même, j'en viendrai à l'ambiguïté de l'approche génétique.

Notre passion de l'avant-texte vise à saisir, dans un fantasme d'exhaustivité et de transparence, rien de moins que le mouvement même de la création. Pour paraphraser une phrase de Claudel sur le XIX ${ }^{\ominus}$ siècle, “[...] On dirait qu'il y a un trésor perdu, voyez [nous] fouillant les archives, déchiffrant des grimoires, remuant la terre, comme les héritiers à la recherche d'un testament [... ${ }^{6}{ }^{6}$ La scientificité des études génétiques est à la fois un instrument et un leurre.

6 Paul Claudel, "Richard Wagner, rêverie d'un poète français», CEuvres en prose, Paris, Gallimard, 1965, p. 865. 
Comme dans toute science, sans doute, il y a encore bien des problèmes insolubles. Le généticien a tendance à systématiser pour instituer un ordre et se rassurer: par exemple, et pour ne pas parler des campagnes de révision si chères à Giovanni Bonaccorso, les stades génétiques, si clairs en théorie, le sont moins dans la pratique, non seulement parce que bien des états de texte sont hétérogènes, mais aussi parce que le degré de schématisation et la place diachronique ne vont pas nécessairement de pair. Un résumé très elliptique peut être ainsi contemporain d'une rédaction élaborée; et des notes placées dans les éditions génétiques, "à l'endroit où l'auteur en a besoin", peuvent avoir été écrites bien avant et faire partie de l'exogénèse. Le généticien peut, en toute rigueur, reconnaître que la délimitation des stades diachroniques n'est pas et ne saurait d'ailleurs être rigoureuse; il peut signaler les lectures conjecturales, les feuillets manquants, les rédactions simultanées et imbriquées, les séries numérales différentes, les problèmes non résolus, il n'empêche qu'en pratique, le lecteur et lui feront comme s'ils n'existaient pas, tant est compulsif notre besoin d'unité.

La complexité même de l'avant-texte flaubertien oblige à des choix problématiques, à des erreurs inévitables qui, par le jeu des éditions, s'enracinent d'autant plus que l'apparat critique est plus rigoureux. Pensez que l'ordre de genèse, quoique dressé à partir d'un recoupement de critères, reste souvent problématique. Sans doute est-ce vrai pour tout texte édité; mais la minutie de l'édition génétique dramatise le problème. Bref, notre science est bien inexacte. Dès lors, ou bien pour nous comme pour Flaubert, la moindre perte, le moindre doute menacent tous les résultats; ou bien l'on s'identifie tellement à Flaubert qu'au contraire, chacun de nos choix devient flaubertien et article de foi.

Car en effet, dans l'exploration de l'avant-texte flaubertien, derrière l'esprit scientifique qui lui sert de caution et lui prête ses instruments, nous guette, par symbiose sans doute, une mystique, la recherche de l'absolu.

C'est le "mysticisme esthétique" 7 de Flaubert; ses rêves d'être "quelque chose d'entier" 8 , un mystique, un brahmane

7 Gustave Flaubert, op. cit., III, p. 16 (A Louise Colet, 1852).

8 Ibid., I, p. 61 (A Ernest Chevalier, 1839). 
perdu dans une absorption démesurée ${ }^{9}$, sa conviction qu'il faudrait tout connaître pour écrire ${ }^{10}$ son rêve d'un style aux phrases «inchangeables" 11 qui serait "une manière absolue de voir les choses". 12

Dans la précision des assemblages, la rareté des éléments, le poli de la surface, l'harmonie de l'ensemble n'y a-t-il pas une espèce de force divine, quelque chose d'éternel comme un principe? ${ }^{13}$

Celui qui suivra Flaubert dans "la folie de la perfection» 14 et les traces du manuscrit devra passer par son mode de vie, sa solitude, sa concentration, son long travail 15 , ses "marinades", ses rêves et ses éblouissements dans les voyages de l'Écriture où "quelque chose d'indéfini vous sépare de votre propre personnalité et vous rive au non-être"16, "ce trou noir étant l'infini même, les rêves se dessinent et passent sur un fond d'ébène immuable. Pas de cris [...] rien que la fixité d'un visage pensif".17 Ceci est du domaine de l'expérience intime, incommunicable. C'est pourtant dans cet itinéraire absolu, audelà des résultats objectifs mais relatifs que chacun lui concède, que l'approche génétique apparaît comme le mode exemplaire des études littéraires.

\footnotetext{
9 Ibid., (À Louise Colet, 1846).

10 Ibid., IV, p. 52 (id. 1854).

11 Ibid., II, p. 468 (id. 1852).

12 Ibid., III, p. 345 (id. 1852).

13 Ibid., VII, p. 294 (A George Sand, 1876).

14 Ibid., Supplt. III, p. 243, (id. 1876).

15 Ibid., II, p. 444 (A Maxime du Camp, 1852).

16 Ibid., III, p. 332, (A Louise Colet, 1853).

17 Ibid., IV, p. 464 (A Mme Roger des Genettes, 1861).
} 